\title{
Combray: Thème du voyage chez Proust
}

Le thème du voyage chez Marcel Proust est 1'un des plus riches et évocateurs de Combray. En effet, dans cette première partie du roman, A la recherche du temps perdu, ce thème se manifeste en plusieurs formes--en voyages littéraux, symboliques, etc.-toutefois reliées d'une antithèse qui les traverse et qui traduit deux désirs opposants: celui de voyager et celui de rester chez soi. 1 Ces formes finissent de si bien s'intercaler que le dessin, même dans les moindres détails, devient presque trop complexe pour le narrateur (et pour nous lecteurs aussi). Par exemple, le nom de l'oeuvre complète, A 1 a recherche du temps perdu, décrit exprès la notion d'une quête; au contraire, Combray, nom spécifique, traduit un endroit particulier où on reste. Pourtant, la synthèse de ces thèmes entremêlés ne se réalisera qu'avec la lecture.

Tout à fait au début du roman, Proust introduit le thème du voyage. Son narrateur, après s'être endormi, se réveille dans 1'obscurité. Il se trouve à mi-chemin entre les mondes de la réalité et du rêve. Sa désorientation subséquente sert de point de départ pour traverser son imagination. Proust, en faisant remarquer que la narrateur se croit "eglise, quatuor, rivalité" (I, 3) souligne trois éléments qui sont respectivement des voyages dans l'espace, dans I'imagination et dans le temps. C'est à dire que son corps se répand pour remplir I'espace d'une église; la musique relève toujours des images, donne un raga indien; et il retourne à une période plus tôt dans le temps avec la rivalité de François Ier et de Charles-Quint. Or, ces voyages sumboliques reflètent un voyage littéral dans la partie du texte qui suit: "relevant les distances" $(I, 3)$. Mais c'est ce qui se passe en 
même temps dans l'imagination du narrateur. Aussi dès le départ du roman avons-nous à faire avec ce jeu de voyages réels et imaginaires.

Le passage cité démontre également pourquoi les voyages sont quelque chose de spécial. C'est grâce

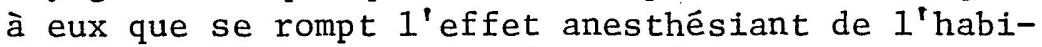
tude; ou, d'autre part, c'est grâce à eux que le narrateur peut parler de "I'excitation qu'il doit à des lieux nouveaux, à des actes inaccoutumés" (I, 3-4). C'est justement le manque de familiarité de nouveaux endroits, cependant, qui peut mener I'inquiétude, la souffrance involontaire et pas voulue. Bien entendu, les choses à qui nous nous sommes habitués sont plus sûres: "car sans I'habitude. . . il serait impuissant à nous rendre un logis habitable" $(I, 8)$.

Pourquoi alors tout risquer pour un voyage? C'est plus facile de rester là, de ne pas en faire l'effort. Tante Léonie est la personnification en chair et en os de cette croyance. Ne voulant pas se fatiguer à l'avance, elle ne quitte pas ses chambres depuis des années, même à la poussée du grand-père du narrateur ( $I, 143)$. Le narrateur même subit cette hésitation lorsqu'il fait face à une tâche difficile: chercher en lui-même pour découvrir ce qui se cache derrière ses impressions $(I, 179)$. Il n'embarquerait pas dans ce voyage sans être rassuré au sujet de ce qui en résulterait. Comme Tante Léonie, il veut plutôt s'en épargner la fatigue.

De plus, dans le voyage littéral d'un malade dont parle le narrateur il s'agit effectivement d'un anti-voyage: ce désir de rester chez soi. Très tôt dans le roman il fait mention d'un "malade qui a été obligé de partir en voyage et a dû coucher dans un hôtel inconnu. . . [mais ] il faudra rester toute la nuit à souffrir sans remède" (I,4). Plusieurs éléments y traduisent le sens d'inquiétude du voyage, le sentiment que ce voyageur aurait préféré rester chez lui. On a d'abord le fait que le voya- 
geur est malade; deuxlèmement, les verbes "obliger" et "devoir" qui impliquent une circonstance contre sa propre volonté; puis l'hôtel "inconnu," adjectif terrifiant au narrateur, pour qui n'importe quel coucher est un drame; et enfin, le verbe "souffrir" qui traduit par sa forme même, plus que le substantif "souffrance" ne peut le traduire, la torture de rester la nuit seul, souffrant.

D'autres voyages littéraux sont évoqués dans le texte: 1e voyage de Paris à Combray chaque saison de Pâques $(I, 48)$ en est un au sens propre du mot. En outre, on y a encore le jeu des deux côtés d'un voyage: le narrateur fait un trajet à Combray. Une fois là, il l'utilise comme point de départ non seulement pour d'autres voyages littêraux et réels (notamment les promenades dont il sera question cidessous) mais encore des objets qu'il connaît pour des voyages symboliques et imaginaires. Une remarque du narrateur est très révélatrice sur ce point-1à: "mon esprit s'agitant pour chercher, sans y réussir, à savoir où j'étais, tout tournait autour de moi dans I'obscurité, les choses, les pays, les années" $(I, 6)$. On constate la progression de substantifs: obscurité, choses, pays, années. L'obscurité signale le départ des lieux accoutumés. Les choses vues ainsi d'une nouvelle manière dans la confusion. perdent leur aspect habituel, ce qui est requis d'un voyage imaginaire; autrement les associations banales ne se laisseraient place aux nouvelles. Sur ce point, on se souvient de 1 'épisode de la petite madeleine $(1,44-48)$. De plus, les choses ouvrent la voie pour la traversée du pays ( 1 'espace) et les années (le temps). C'est exactement ce que veut dire le narrateur quand il parle de 1'homme qui dort: "le fauteuil magique le fera voyager à toute vitesse dans le temps et dans l'espace" $(I, 5)$. Encore des exemples: grâce à la métempsycose, le narrateur prend un voyage qui symbolise l'évolution de l'être humain. "L'homme des cavernes" $(I, 5)$ révèle son

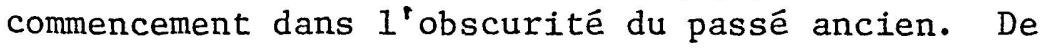


là, il passe en une seconde des images de choses (lampes à petroles, chemise à col rabattu) et le souvenir d'où il aurait pu être (I'espace, l'endroit) pour continuer, jusqu'à présent, "par-dessus des siècles de civilisation" (temps!) (I, 5-6). Le narrateur reconnaît également que les vieilles choses d'une chambre qui lui donne "Ia nostalgie d'impossibles voyages dans le temps" (I, 41), impossibles au sens littéral, non pas imaginaire. Les voyages imaginaires et symboliques deviennent donc en quelque cas plus importants, plus magiques, même plus satisfaisants que ceux qui sont littéraux.

Après avoir découvert cette vérité essentielle, 1 'occasion de faire de tels voyages se montre même dans les moindres détails de la vie quotidienne, tels que 1 'habitude de manger les aspèrges. Des aspèrges? --des choses tout à fait' banales! Cependant, à partir d'elles le narrateur s'enlève vivement d'abord à 1'océan ("trempées d'outre-mer," I, 121) et dans l'au-delà ("irisations qui ne sont pas de la terre," I, 121). I1 s'agit ici des voyages extraordinaires dans l'espace après lesquels il redescend en glissant 1'arc-an-ciel pour retourner chez lui (I, 121). Or, il part pour voyager avec Golo et Geneviève de Brabant au sixième siècle. C'est un voyage réalisé par les irisations évocatrices de la lanterne magique. ( I, 9-10)

L'église de Combray résume toute cette idée. $N^{\prime}$ importe quel aspect de la France--vieux pays parsemé de monuments anciens et de traditions même plus anciennes--peut nous enlever à un voyage dans $1 \mathrm{e}$ passé: "déployant à travers les siècles son vaisseau qui semblait vaincre et franchir des époques successives d'où il sortait victorieux" (I, 61). Or, 1 'église qui voyage symboliquement dans le passé incorporé dans son présent ne se comprend que par les limites matérielles de ses pierres de taille. De la même manière le narrateur qui traverse les royaumes de son soi (auquel figurent le passé et le présent) n'est restreint que par sa volonté d'y cher- 
cher. Ainsi ses souvenirs--les vestiges du passé-sont-ils 1 'équivalent des parties de I'église survécues des époques différentes. Et comme l'ëglise qui a traversé le temps, 1 'exaltation du narrateur a porté ses souvenirs et "a réussi à leur faire traverser tant d'années successives" ( $I, 184$ ).

L'épisode de la petite madeleine, lorsque le narrateur essaie de trouver une raison derrière sa joie, souligne surtout ce voyage symbolique en luimême. Bien sûr, ce n'est pas sans quelques hésitations, quelques désirs de "rester chez soi" à la surface de son esprit, quelques soucis pour ne pas se fatiguer à 1 'avance. De plus, il éprouve la "Grave incertitude. . . quand lui, le chercheur, est tout ensemble le pays obscur où il doit chercher" (I, 45), Mais il se rend enfin compte que la quête vaut l'effort. Le narrateur se fait donc voyageur; il entend "la rumeur des distances traversées" ( $\mathrm{I}, 46)$; il réussit à arriver à sa destination. On se rend mieux compte à quel point la petite madeleine lui est significative.

Le voyage en lui-même et le voyage littéral se trouvent face à face dans les promenades aux deux côtés de Combray: celui de Guermantes et celui de Méséglise. Ils représentent deux voyages séparés dans la pensée du narrateur. On les commence aux portes différentes, et on a cette "habitude de n'aller jamais vers les deux côtés un même jour" ( $I, 135)$. Chaque chemin a ses aspects particuliers, n'associés qu'avec lui. Le narrateur décrit le côté de Méséglise comme "I'idéal de la vue de plaine" (I, 135) où on voit la pluie, les aubépines, Gilberte et Swann. Du coté de Guermantes c'est "I'idéal du paysage de rivière" (I, 135) où se trouve la Vivonne, les nymphéas, le pêcheur inconnu, la Duchesse de Guermantes. Mais plus révélateur, le narrateur considère le côté de Méséglise comme quelque chose de réel mais "inaccessible comme 1 'horizon" ( $I, 134$ ) tandis que celui de Guermantes est "le terme plutôt idéal que réel de son propre côté" (I, 134). 
Le problème, c'est que, pour Iui, les côtés Méséglise et Guermantes symbolisent deux voies de la vie tout à fait séparées. Le narrateur fait des expectatives basées sur les côtés et distingue donc leurs fins. Le côté de Méséglise est celui de voyages symboliques en lui-mêrme. Ce sont les réalités du désir, du plaisir, de l'amour: le désir d'une femme, le plaisir pervers de Mlle Vinteuil, I'amour idéal du narrateur pour Gilberte et $I^{\prime}$ amour raté de Swann pour Odette. C'est la route inconnue en lui-même de la masturbation et celle où il est "frappé pour la première fois de ce désaccord entre nos impressions et leur expression habituelle" ( $I, 155)$.

Du côté de Guermantes, "le côté ensoleillé," il découvre son désir d'être écrivain, mais il craint que ce ne soit un but idéal qu'il n'atteindra pas, de même qu'on n'atteint jamais Guermantes ( $I, 171)$. Aussi est-ce encore l'hésitation d'un voyageur, le sentiment de " 1 'anti-voyage" qu'il y éprouve. Mais c'est aussi le côté du pêcheur qu'il ne connaît pas. Ce pêcheur qui, comme indicateur, signale le début d'un chemin et offre l'invitation au voyage à la fois. C'est un chemin éventuel qui s'évoque encore de ce côté par les clochers de Martinville. Ces clochers, dont les tours voyagent sur l'horizon, jouent un rôle capital dans la vie du narrateur. Ils font remuer les premières poussées de son esprit littéraire, ce qui s'éclate tout d'un coup dans des mots écrits ( $I$, 180-82). Comme il 1'a déjà fait du côté de Méséglise, il découvre du côté de Guermantes une autre vérité de lui-même: il existe "ces états qui se succèdent" en lui ( $I, 183$ ).

Les côtés, tons les deux, aboutissent donc aux découvertes de la Vérité." Ce n'est pas la vérité philosophique mais celle de son soi et de sa vie. Et en voyant aussi à quel point ces deux côtés sont reflétés dans tous les aspects de sa vie même, le narrateur découvre qu'il ne faut pas les séparer, ou on en serait déçu: "comme ceux qui partent en voyage pour voir de leurs yeux une cité désirée et s'ima- 
ginent qu'on peut goûter dans une réalité le charme du songe". ( $I, 5)$. Or, il faut faire la synthèse des côtés réels et iréels, synthèse qui se réalise quand le narrateur rencontre la Duchesse de Guermantes dans la chapelle de l'église. Là il doit faire face à ses expectatives et à leur réalité. Autrement dit, les ressemblances entre les promenades des deux côtés sont frappantes (malgré la croyance au contraire du jeune narrateur) et font ainsi allusion à une synthèse future de sa part. Par exemple, l'allée sur la propriété de Swann rappelle le cours de la Vivonne "dont 1'accès était ouvert au publique" ( $I, 169)$ de même qu'une "pièce d'eau. . . creusée" (I, 136) fait allusion à des "travaux d'horticulture aquatique" (I, 169) ainsi que "I'étang artificiel" (I,135) et "les petits étangs que forme la Vivonne" (I, 169). Mais le rapprochement va plus loin. "Les Nymphes du printemps" ( $I, 136)$ dans le jardin français du côtê de Méséglise évoquent le jardin des Nymphéas du côté de Guermantes et les images qu'elles relèvent ( $I, 169)$. De plus, les "grand arbres". (I, 136) du côté de Méséglise suggèrent "les rives. . . très boisées" (I, 169) de la Vivonne; les belles aubépines roses se comparent aux nymphéas à coeur écarlate; et "les fleurs. - comme des pompons qui enguirlandent une houlette rococo" ( $I, 139$ ) relèvent des guirlandes d'une fête galante à la Watteau (I, 169). Et à tous les deux endroits le narrateur éprouve vivement le désir de rester là pour en jouir, mais il est contenu par le flot qui comprend aussi bien le temps que le voyage. Du reste, ses parents l'appellent à continuer le "voyage": : "il me fallait rejoindre en courant mon père" ( $I, 138)$ et "si Virgile, s'éloignant à grands pas, n'avait forcé à le rattraper au plus vite, comme moi mes parents" (I, 169).

Les deux côtés ne sont pas donc complètement séparés comme le narrateur les décrit. Ils sont plutôt réliês à une même vérité, tant qu'il y a une clef dans tout ce jeu: la synthèse des deux dans la littérature d'abord au moyen de la lecture et puis 
au moyen de 1'écriture. Ainsi se fait un rapprochement des voyages littéraux (comme ceux de chaque côté de Combray) et du progrès de l'esprit: "Aussi le côté de Méséglise et le côté de Guermente se restent-ils pour moi liés à bien des petits événements de celle de toutes les diverses vies que nous menons parallèlement, qui est la plus pleine de péripéties, la plus riche en épisode, je veux dire la vie intellectuelle" ( $I, 183)$.

En outre, ce sont "les vérités [sur lesquelles 1a vie intellectuelle s'est construite] qui nous ont ouvert de nouveaux chemins, nous en préparions depuis longtemps la découverte" (I, 183). Et, pour le narrateur, les vérités trouvées au long du chemin dans la réalité (soit en promenade, soit en voyages littéraux) aussi bien que les vérités découvertes à l'instar de l'irréalité (voyages imaginaires, symboliques) se trouvent toutes dans la lecture. C'est là où on goûte les plaisirs d'y rester et lire. C'est dans la lecture que $I^{\prime}$ 'on peut découvrir de nouvelles idées et voyager dansl'esprit. Ainsi s'explique le narrateur: "j'ai eu, à cause du livre que je lisais alors, la nostalgie d'un pays montueux et fluviatile" (I, 86). Aussi, pendant qu'on fait voyage de 1 'esprit en lisant se rend-on compte que le voyage continue toute la vie. Puisqu'un voyageur apprécie des choses avec des yeux frais, inaccoutumés, ses expériences comme voyageur dans la littérature peuvent aboutir à une nouvelle vision. Du moyen de cette vision il apprécie même plus les choses déjà autour de 1ui. Sur ce point, le narrateur dit que "ces paysages des livres que je lisais n'étaient pas pour moi que des paysages plus vivement représentés à mon imagination que ceux que Combray mettait sous mes yeux" ( $I, 86)$.

Le cercle est donc complet. Du fait qu'en lisent, on voyage, ce qui mène à la découverte de nouvelles vérités. Et le sommet de cette perception ne vient au narrateur que dans 1 'épisode des deux clochers de Martinville. Le narrateur imprime pour 
toujours les vérités derrière sa joie sur ces images relevées. Ses mots écrits deviennent contenus et contenants en ce qu'ils contiennent: ils sont des images flottantes qui ont voyagé depuis la profondeur de son soi à leur expression à la surface de son esprit. Contenus par le flot du temps, mis à repos, ils voyageront à jamais, une petite boule de sa vérité.

\author{
CYNTHIA A. FROGLEY \\ UNIVERSITY OF KANSAS
}

NOTE

1 Marcel Proust, A 1a recherche du temps perdu, édition établie et annotée par Pierre Clarac et André Ferré, 3 vols. (Paris: Gallimard, 1954). Toutes les citations renvoient à cette édition. 


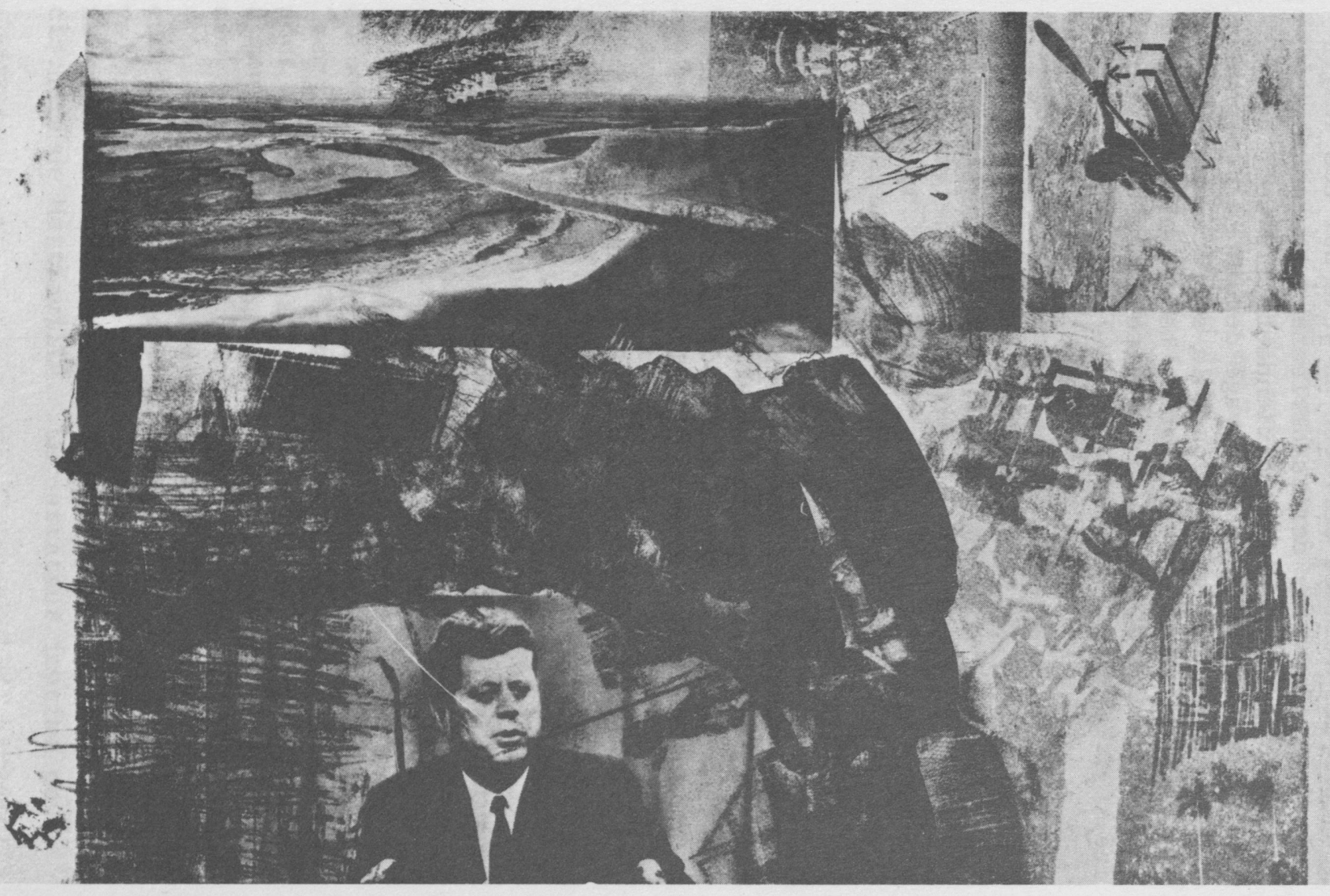

